\title{
A new method to elucidate fracture mechanism and microstructure evolution in titanium during dissimilar friction stir welding of aluminum and titanium
}

\author{
Amlan Kar ${ }^{\mathrm{a}, \mathrm{c}, *}$, Sergey Malopheyev ${ }^{\mathrm{b}}$, Sergey Mironov ${ }^{\mathrm{b}}$, Rustam Kaibyshev ${ }^{\mathrm{b}}$, Satyam Suwas ${ }^{\mathrm{c}}$, \\ Satish V. Kailas ${ }^{\mathrm{c}}$
}

${ }^{a}$ Indian Institute of Technology (Indian School of Mines), Dhanbad, India

${ }^{\mathrm{b}}$ Belgorod National Research University, Belgorod 308015, Russia

${ }^{\mathrm{c}}$ Indian Institute of Science (IISc), Bengaluru 560012, India

\section{A R T I C L E I N F O}

\section{Keywords:}

Friction stir welding

Dissimilar materials

Particles

Microstructure evolution

Recrystallization

Adiabatic shear band

\begin{abstract}
A B S T R A C T
In the friction stir welding (FSW) of dissimilar materials, the weld nugget exhibits composite properties and is composed of hard particles (high-strength material) distributed in a soft matrix material. The distribution of these particles influences the properties of the weld. Therefore, it is useful to characterize the deformation and fragmentation of the high-strength material from which they originate. In the current study, FSW of aluminum (Al) to titanium ( $\mathrm{Ti}$ ) was performed and a new technique was introduced to remove $\mathrm{Al}$ from the post-weld sample to characterize the deformation and fragmentation of $\mathrm{Ti}$ in the weld nugget. The post-weld sample showed that Ti particles were inhomogeneously distributed. It was understood that the plastic deformation of the Ti depends on its location of the weld. A detailed investigation revealed a number of fracture zones in titanium. A systematic analysis revealed the evolution of twins in $\mathrm{Ti}$ in the thermos-mechanically affected zone (TMAZ), whereas notwins and recrystallized grains were noticed on the Ti flake that was consolidated in the weld nugget. The Xray computed tomography (XCT) showed that Ti flakes were subjected to deformation and bending. Furthermore, it was noticed that the width of the flakes was not identical along their length and that the flakes were broken at the middle region into two parts. It can be concluded that $\mathrm{Ti}$, at the interface and as flakes, was subjected to severe deformation at a lower homologous temperature that leads to the formation of adiabatic shear bands (ASBs). The local evolution of temperature within the ASBs was much higher than the general weld temperature, leading to the recrystallization of $\mathrm{Ti}$ at the interface. Moreover, ASBs are prone to crack nucleation and propagation; hence, particles with different morphology and sizes were noticed in the nugget zone. The distribution of particles was inhomogeneous due to variation in the particle size and complex mechanical mixing. The distribution of particles varied with location across the weld nugget. A homogeneous mechanical mixing was noticed at the center of the weld nugget due to complex material flow and distribution of finer particles. With this understanding of the deformation and fracture mechanism of titanium during dissimilar $\mathrm{FSW}$ of $\mathrm{Al}$ to $\mathrm{Ti}$, it is possible to engineer the developed composites and/or weld nuggets of dissimilar materials to achieve the required combination of mechanical properties.
\end{abstract}

\section{Introduction}

New and efficient designs of engineering components are being developed and many structures are composed of more than two materials that need to be joined by welding. The welding of titanium to aluminum has significant application in aerospace components where high specific strength of a structure is a key prerequisite for greater fuel efficiency. The joining of different materials by the advanced and conventional fusion welding processes is a challenge owing to the dissimilarities in their metallurgical and mechanical properties. Further, the evolution of brittle intermetallics in dissimilar welds causes a reduction in performance and efficiency [1]. Moreover, poor weldability and the presence of solidification-related defects restrict the application of fusion welding for certain materials like aluminum.

\footnotetext{
* Corresponding author at: Indian Institute of Technology (Indian School of Mines), Dhanbad, India.

E-mail address: amlankar@iitism.ac.in (A. Kar).
} 
FSW is considered a solid state welding process where the asreceived materials are subjected to thermos-mechanical deformation. It is likely to provide a solution to the problems associated with fusion welding. In FSW, the combined effect of the friction and severe plastic deformation results in temperature rise that leads to joining of materials [2]. The heat generated during friction softens the material and it eventually gets displaced from the leading edge to the trailing edge; it consolidates at the trailing edge by a forging force due to the tilt angle of the tool. In the recent past, FSW was introduced in dissimilar welds to restrict the formation of cavities and intermetallics due to mechanical stirring in the weld nugget [3,4]. Defects are formed in dissimilar FSW due to the selection of improper process parameters and inappropriate mechanical mixing [5]. Kar et al. [6] showed that the tool offset influenced ternary mechanical mixing and formation of voids in the dissimilar weld of pure $\mathrm{Al}$ to Ti with a copper interlayer. Kumar et al. [7] found out that the quality of mixing was asymmetric across the faying interface; optimum mixing was achieved when the faying interface was on the advancing side (AS).

In the mechanically mixed zone, the size of the particles and their distribution can have a decisive impact on the microstructure evolution and tensile properties of the weld [8], which would behave like a composite [9]. The property of a composite varies with the distribution, agglomeration and size of the second phase hard particles in a soft matrix. These issues can be addressed by FSW and friction stir processing (FSP) $[10,11]$. Madhu et al. [12] used un-annealed titanium dioxide $\left(\mathrm{TiO}_{2}\right)$ particles to prepare an $\mathrm{Al}-\mathrm{TiO}_{2}$ nano-composite using FSP. The authors showed that the $\mathrm{TiO}_{2}$ particles $(\sim 1 \mu \mathrm{m})$ get dispersed in an aluminum matrix through six passes of processing. The coarse $\mathrm{TiO}_{2}$ particles fractured during these multiple passes, which resulted in the distribution of nano-sized particles (size $<100 \mathrm{~nm}$ ) in the Al-matrix. The fragmentation of particles and their homogenous dispersion lead to the formation of a nano-composite with superior mechanical properties.

The mechanisms leading to such improvement in mechanical properties can be demarcated into direct (load sharing with particles) $[13,14]$ and indirect strengthening (changes in microstructure and its evolution in the presence of particles) [15,[19]]. Kar et al. [13] illustrated the importance of mechanical mixing of materials, formation intercalated particles and distribution of finer particles to obtain dissimilar weld with higher mechanical properties. Therefore, it is very important to investigate the spatial distribution of Ti particles in the Al matrix (mechanical mixing/materials mixing) in the weld nugget during dissimilar friction stir welding in order to further enhance the property. However, there is limited literature reporting the deformation mechanism and in-situ formation of Ti particles during dissimilar FSW. Hence, it is imperative to investigate the mechanism of formation of Ti particles during FSW in order to optimize the process conditions to achieve a good weld.

The shear deformation during FSW aids in the deformation and fragmentation of $\mathrm{Ti}$ on the advancing side. It leads to the formation of particles of different sizes. Kar et al. [13] performed a two-pass FSW of Al alloy to Ti alloy to investigate the effect of the second pass on the deformation mechanism of Ti particles, microstructure development and tensile properties of the weld. It was predicted, from the systematic microstructural investigation of $\mathrm{Ti}$, that the continuous fragmentation in $\mathrm{Ti}$ particles took place by the formation of micro-cracks due to the evolution of shear bands. Some identical results have been reported in the literature during the friction stir welding of $\mathrm{Al}$ to $\mathrm{Ti}$ [17]. However, the evolution of shear band formation in Ti has not been identified from the microstructural characterization. All the studies mentioned above have focused on the intermetallic phase formation, microstructural development and final tensile properties of the weld. The number of reports on the mechanism of fragmentation and deformation behavior of the titanium in the nugget zone are limited. Such a study is important as this sill decide the properties of the weld.

This paper reports the results of a fundamental study on the process during the FSW of $\mathrm{Al}$ to $\mathrm{Ti}$. The cross-section of the weld was characterized to predict the distribution of Ti particles in the $\mathrm{Al}$ matrix. To elucidate the mechanism of particle formation, the Al was externally dissolved from the post-weld sample to characterize the fragmentation of Ti during welding. A systematic study was performed on the Ti side of the weld and on the Ti flake to explore the deformation mechanism that led to the formation of particles and flake. Based on the post-mortem examination of welds, the mechanisms involved in the joint formation is also discussed in this paper.

\section{Materials and methods}

\subsection{Materials and processing}

Commercially procured rolled plates of pure, $4 \mathrm{~mm}$ thick aluminum (Al) and titanium (Ti) were selected for the dissimilar weld. The plates were cut as per the required dimension $(200 \mathrm{~mm} \times 75 \mathrm{~mm} \times 4 \mathrm{~mm})$ and subjected to friction stir welding using a custom-built 2-axis FSW machine (developed with the help of IISc, Bangalore and ETA (P) Ltd., Bangalore) in a butt-joint configuration, as shown in Fig. 1. A cylindrical tool made of WC-8\%Co alloy with a shoulder diameter of $20 \mathrm{~mm}$ was used for welding. The length and diameter of the tool pin were $3.5 \mathrm{~mm}$ and $4.0 \mathrm{~mm}$, respectively. A side chamfer of $1.0 \mathrm{~mm}$ was provided on the shoulder and edges of pin to minimize the possible formation of crack (fracture) on the edge of the tool while in motion. During FSW, the rotational tool speed, traverse speed, plunge depth, tool offset were chosen as $800 \mathrm{rpm}, 40 \mathrm{~mm} / \mathrm{min}, 3.7 \mathrm{~mm}$ and $1.5 \mathrm{~mm}$ on the $\mathrm{Al}$ side from the faying interface, respectively. The offset of $1.5 \mathrm{~mm}$ was chosen to get titanium flake (large and elongated titanium particle) in the weld nugget as a tool offset of $2 \mathrm{~mm}$ led to formation of fine particles instead of titanium flake. Unlike titanium particles, it is comparatively convenient to electropolish and chemical etching of titanium flake (deposited in the weld nugget) for microstructural characterization. During welding, tool tilt angle was set as $2.5^{\circ}$ that was kept constant all through the weld.

\subsection{X-ray micro-CT scan}

X-ray Computed Tomography (XCT) is a three dimensional (3D) imaging procedure that uses many $\mathrm{X}$-rays measurements taken from different angles to create a volumetric image of a physical object using the Zeiss Versa 520 system. It allows the user to locate some particular elements inside the object without destroying or cutting the multicomponent and porous samples. Using computer-processed algorithem, it is primarily used to visualize the distribution of each element and quantify them in the dissimilar welds. The geometric positioning of the object with respect to the source and detector yielded a resolution of $8 \mu \mathrm{m}$ for the sample.

\subsection{Microstructural characterization}

To examine the microstructure of the weld, the specimens were cut using Wire Electrical Discharge Machining (WEDM) perpendicular to the direction of welding. The weld specimens were mechanically polished using Emery paper and cloth polishing by standard metallographic methods (to remove the deformed layer formed due to WEDM). The mirror-finished samples were etched with Keller's reagent for $45 \mathrm{~s}$. To characterize the deformation and fragmentation of $\mathrm{Ti}$, the $\mathrm{Al}$ was externally dissolved at room temperature for $24 \mathrm{~h}$ from the post-weld sample using aqueous sodium hydroxide $(\mathrm{NaOH} ; 25 \%$ concentration) solution ( [18]. During the dissolution of Al, the chemical reaction leads to formation of sodium aluminate $\left(\mathrm{NaAlO}_{2}\right)$ salt and hydrogen gas. The aim in selecting the duration of the reaction time was that the $\mathrm{Al}$ in the samples should completely be consumed during the reaction. To be confirm about the complete dissolution of $\mathrm{Al}$ from the weld sample, the color of the sample was monitored as the reaction proceeds. This color turned into dark gray as the metal was completely consumed after $24 \mathrm{~h}$, which signified the complete dissolution of Al. The residue, which is 


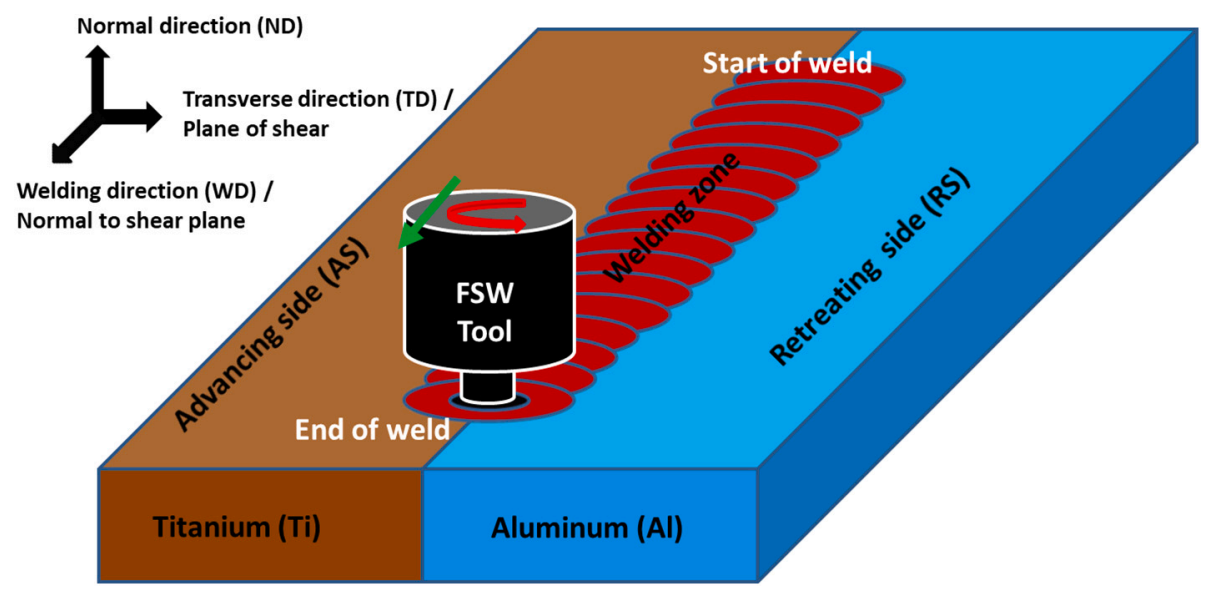

Fig. 1. A schematic representation of experimental arrangement and location of the different process zones in the weld.

solid substances (fine titanium particles and Ti weld zone) and an aqueous solution of $\mathrm{NaAlO}_{2}$, is then separated from the solution using filter paper. This process was used to separate the solid Ti from the liquid solution. Kar et al. [17,19] proposed that these fine particles were either intercalated or intermetallics in composition and hence, chemical reaction and corrosion during dissolution of $\mathrm{Al}$ made these particles difficult for microstructural characterization and chemical analysis. The unreacted sample was examined by an Optical Microscope (OM) and a Scanning Electron Microscope (SEM). In order to assess the finer microstructural details, Electron Backscatter Diffraction (EBSD) was used. For this study, the Ti sample from the AS of the weld was electropolished with standard A3 electrolyles using voltage and time of $36 \mathrm{~V}$ and $10 \mathrm{~s}$, respectively. The EBSD scans were performed using the TSL (Tex Sem Lab) data acquisition system fitted with a tungsten-filament SEM and the data analysis was done using the TSL-OIM software. The EBSD data are represented in the form of inverse pole figure (IPF) maps that show the position of a sample direction relative to the crystal reference frame. Grain Boundary Character Distribution (GBCD) map exhibits three types of grain boundaries. They are sub-grain boundaries (SGB), the low-angle grain boundaries (LAGB) and high-angle grain boundaries (HAGB) across which misorientation varies from 2 to $5^{\circ}$ (represented by red color), $5-15^{\circ}$ (represented by green color) and $15-180^{\circ}$ (represented by blue color), respectively. In addition, Grain Orientation Map (GOS) and Kernel Average Misorientation (KAM) map were constructed to characterize the residue deformation (local and global) inside the grains of a recorded microstructure. The remnant deformation is due to the gathering of dislocation. This is used to demarcate recrystallized grains and plastically deformed grains with the help of GOS map. A lower GOS value (GOS $\leq 2)$ point to recrystallized grain, whereas higher GOS value (GOS $>2$ ) illustrates the accumulation of dislocation due to plastic deformation. In contrast, the KAM map was generated to demonstrate the accumulation of local strain in each pixel of the map. This map gives information about the degree of local orientation mismatch due to dislocation density.

\section{Results}

\subsection{Optical image analysis}

The representative macrostructural features of the cross-section of the weld is shown in Fig. 2(a). Large Ti flakes were observed in the weld nugget. In addition, a zone with distribution of fine $\mathrm{Ti}$ particles was visible (indicated with a white circle) in between the Ti flakes and faying interface,. The Ti particles were homogeneously distributed in the weld nugget. On the other hand, the flakes were consolidated on the retreating side (RS) of the weld and were connected to the joint Ti interface only at the bottom of the weld. Moreover, no welding defect was noticed in the weld nugget and at the joint interface. To characterize the deformation and fragmentation of $\mathrm{Ti}$ alone, $\mathrm{Al}$ was dissolved by submerging the weld into a sodium hydroxide $(\mathrm{NaOH})$ solution. The top view of the sample after dissolving $\mathrm{Al}$ is shown in Fig. 2(b). The deformation (by the tool pin) region of Ti was noticed at the bottom-center of the weld. In this zone, a continuous ring pattern was visible. In addition, a small incompletely deformed Ti region (marked with a white circle in Fig. 2(b)) was noticed in the center zone. A similar deformation pattern was seen in the top region of the sample where deformation of titanium had taken place due to its interaction with the tool shoulder (marked with a white square in Fig. 2(b)). In the cross-section of the same sample (shown in Fig. 2(c)), it was seen that the width of the flakes varies with location; the width was higher at the top of the weld than at the bottom (marked with arrows in Fig. 2(c)).

\subsection{Scanning electron microscopy}

The detailed interfacial features of the sample, after dissolving aluminum, were examined using SEM. Fig. 3(a) shows the secondary electron (SE)-SEM micrograph of the weld. The bottom of the weld exhibits a microstructure with substantial deformation in comparison to the top of the weld. A detailed SEM micrograph of the top of the weld is shown in Fig. 3(b). A number of fracture zones were noticed. It can be seen from the Fig. 3(b) that a specific particle has not undergone an equivalent degree of deformation compared to those on its two sides, resulting in variation in deformation and fragmentation. A similar deformation pattern was noticed at the bottom of the weld. A number of crack paths were noticed in Fig. 3(c). All the crack paths were interconnected and crack branching was also seen. The Fig. 3(d) shows an SEM micrograph from the interface of the flake. A number of parallel fracture lines were observed. The lines were oriented along the direction of welding and hence, they resembled the deformation direction during welding. Fig. 3(e) shows a particle at the bottom region of the weld. The particle exhibited a different morphology when compared to the zone outside it. The small void that appeared in the weld could be due to mechanical mixing and the corresponding dissolution of Al. A similar zone, along with fragmentation of titanium, was noticed at the bottom ring zone (as shown in Fig. 3(f)). This indicates severe fragmentation of $\mathrm{Ti}$, followed by inhomogeneous mixing of Ti particles with $\mathrm{Al}$.

\subsection{Electron backscatter diffraction}

For an in-depth understanding of the mechanism behind the microstructural evolution of titanium, electron backscatter diffraction (EBSD) scans were carried out in different regions such as: (i) Ti away from the joint interface and (ii) Ti flake that was deposited in the weld nugget after getting deformed and separated from the $\mathrm{Ti}$ interface. It is 


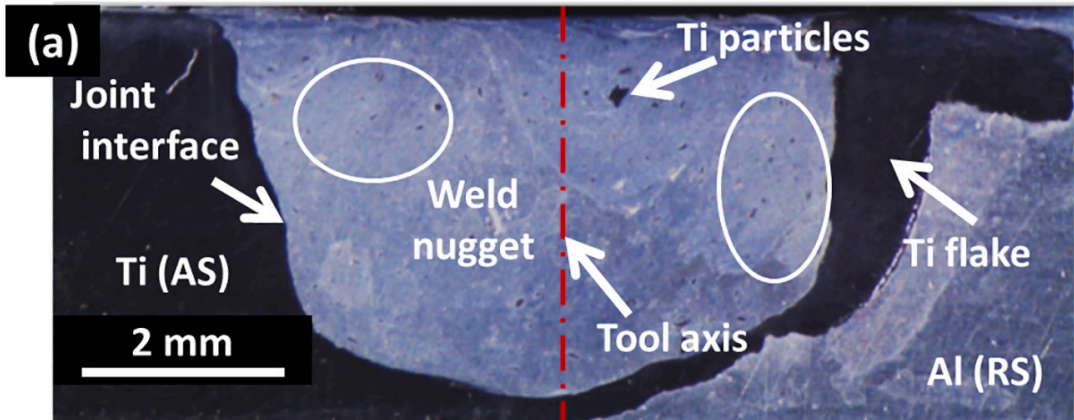

TD $\bigwedge_{\text {WD }}^{\text {ND }}$

WD $=$ Welding direction $/$ Normal

to shear plane

TD = Transverse direction/ Plane

of shear

ND $=$ Normal direction
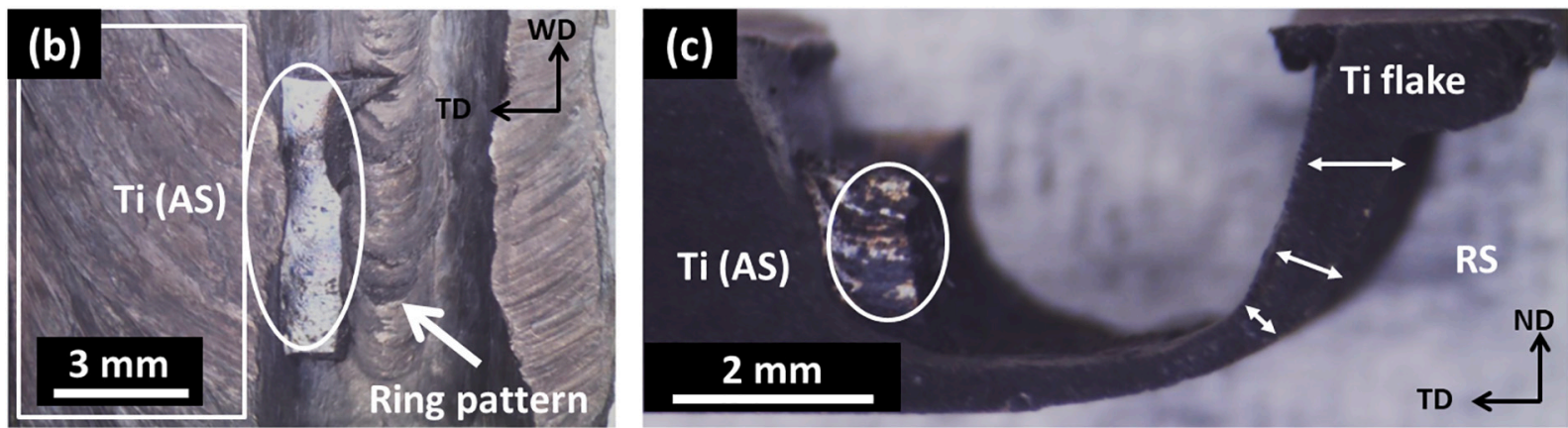

Fig. 2. Optical micrographs of $\mathrm{Al} / \mathrm{Ti} \mathrm{FSW}$ sample after dissolution of $\mathrm{Al}$; a) side view (cross-section) and b) top view showing deformation of Ti flake after the weld. c) shows the only $\mathrm{Ti}$ structure after the dissolution of $\mathrm{Al}$ by a solution of $\mathrm{NaOH}$.

important to note that these two regions are subjected to different levels of strain and temperature, which steadily increase from the as-received $\mathrm{Ti}$ to the weld interface where the strain-hardening processes due to severe deformation predominate the softening ones owing to rise in temperature [20]. It is important to note here that The microstructure of $\mathrm{Ti}$ base material comprises randomly oriented equiaxed grains with an average grain size of around $40 \mu \mathrm{m}$ and it contains very small fraction of deformation twins. The EBSD data associated with Ti base material has been presented by Kar et al. [17].

Therefore, a systematic study could lead to elucidate the mechanism of microstructure development and the possible reason for formation of Ti particles and flakes, as discussed by Kar et al. [17]. Fig. 4(a) shows the
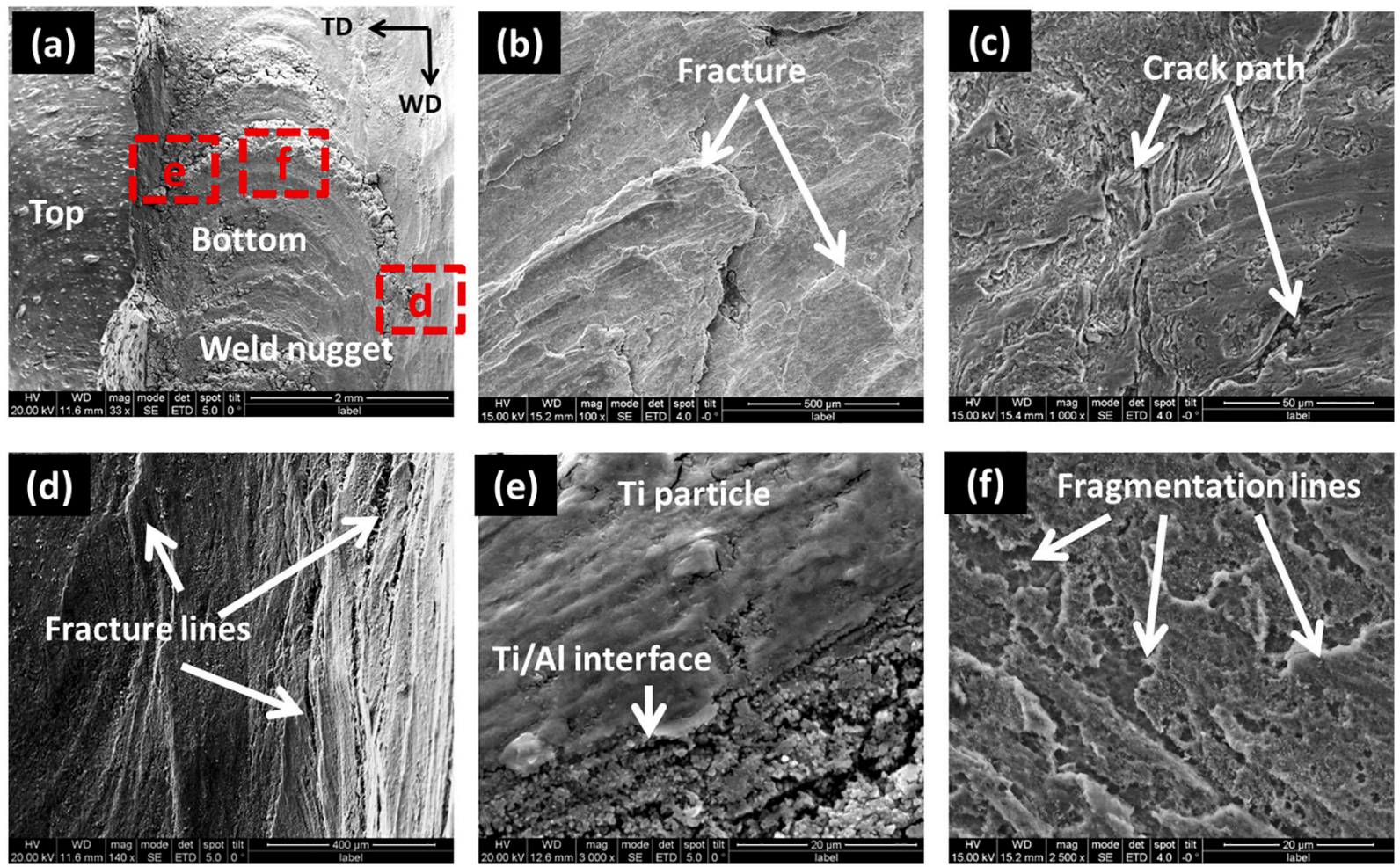

Fig. 3. SEM micrographs of the weld after dissolving Al; a) macro-image of the deformed Ti in the weld nugget, b) fracture in Ti in the shoulder-affected zone at the top side of the weld, c) crack propagation path in $\mathrm{Ti}$, d) parallel fracture line at the joint interface, e) Ti particle and $\mathrm{Al} / \mathrm{Ti}$ interface at the bottom of the weld nugget and f) complicated fragmentation lines at the bottom of the weld nugget. 
IPF map of Ti that is close to the TMAZ. The IPF map illustrated the evolution of grains of different sizes and shapes. The microstructure of $\mathrm{Ti}$ consisted of grains with an average grain size of around $11 \pm 3 \mu \mathrm{m}$, which were randomly oriented and distributed. A number of elongated grains with high aspect ratios (marked with black arrows) were also noticed. There are two boundaries between the said region and the original grain. The GBCD map, as depicted in Fig. 4(b), indicates a HAGB between the elongated grain and the original grain. These regions are termed as twins. Further, it was noticed that the microstructure of Ti was comprised of a low fraction (25.4\%) of HAGBs. The GOS and KAM maps from the microstructure are shown in Fig. 4(c) and Fig. 4(d), respectively. It can be seen that there was no accumulation of the local orientation gradient and microstructure contains a very low fraction of dislocation density. Moreover, Fig. 4(d) indicates $12 \%$ recrystallized grains ( $\mathrm{GOS}=0-2^{\circ}$ ) in the microstructure that have originated locally at the junctions of HAGBs.

The EBSD scan corresponding to the titanium flakes that are consolidated in the nugget zone is shown in Fig. 5. Fig. 5(a) displays the IPF map that exhibited a random grain orientation. A remarkable reduction in the average grain size was observed in the grains from Fig. 5 (a) to Fig. 4(a): from $40 \mu \mathrm{m}$ to $8 \mu \mathrm{m}$. A few coarse grains were noticed in the microstructure. In addition, a high fraction of twins and fine grains were seen in the IPF map. These grains primarily originated at the boundaries of original grains and twins. This indicates the evolution of twins and fine grains from the as-received coarse grain structure, as seen in Fig. 4(a). The GBCD map (shown in Fig. 5(b)) exhibits an increase in the fraction $(50 \%)$ of HAGBs. This fraction was higher than that observed in titanium in the TMAZ (Fig. 4(b)). The KAM map (Fig. 5(c)) shows a significant reduction in dislocation density. The GOS map (Fig. 5(d)) reveals a substantial increase (71\%) of recrystallized grains. This fraction of recrystallization was higher than that observed in Fig. 4 (d) in spite of the increase in plastic deformation and temperature rise in the weld nugget. This indicates an alternative mechanism of recrystallization in $\mathrm{Ti}$ instead of conventional static and dynamic
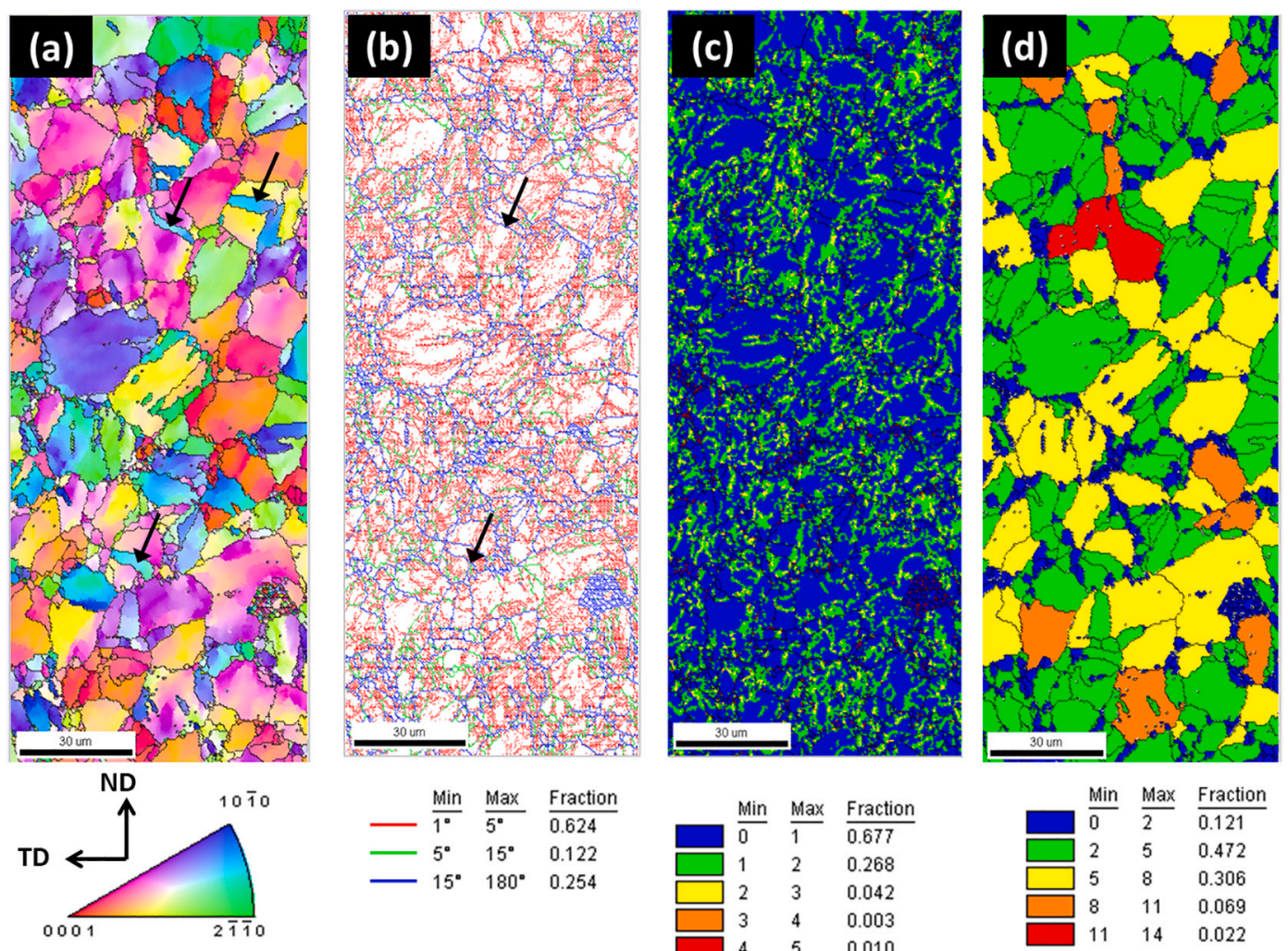

recrystallization.

\subsection{X-ray computed tomography}

Since titanium and aluminum possess different linear attenuation coefficients, the XCT is used to visualize the distribution of different phases [21], such as $\mathrm{Ti}$ and $\mathrm{Al}$, in the scanned area of the weld sample. For example, in the FSW of $\mathrm{Al}$ to $\mathrm{Ti}$ with a niobium (Nb) interlayer, Kar et al. [4] used XCT techniques to demarcate the distribution and morphology of $\mathrm{Ti}$ and $\mathrm{Nb}$. By selecting a user-defined threshold range that belongs to $\mathrm{Ti}$ only, the location of $\mathrm{Ti}$ (with a different morphology) in the weld nugget was studied in the present investigation. Fig. 6(a) shows a three-dimensional (3D) XCT image that was taken from the weld nugget. From these images, it can be seen that the Ti flake was subjected to deformation and bending in the weld nugget. Fig. 6(b) shows a 3D top view of the same image revealing two elongated flakes that were adhered to the large flake and joint interface. A precise observation revealed that the widths of the flakes are not identical along their length and that the flakes were broken at the middle region into two parts. Interestingly, a hollow region was visible near the center-bottom region of the weld. This appeared due to the mixing between $\mathrm{Al}$ and $\mathrm{Ti}$, which virtually disappeared from the image because only Ti was selected to reconstruct the image and the density of the mixing zone and intercalated particles were substantially different from that of pure Ti.

\section{Discussion}

\subsection{Microstructural evolution and fragmentation of titanium}

In FSW, the relative rotation and corresponding interfacial friction between the tool and workpiece at high strain and strain rates increase the weld temperature and plastic deformation of the material. This leads to an increase in temperature (peak temperature) at the center of the weld and causes heat conduction from center of the weld nugget to the 

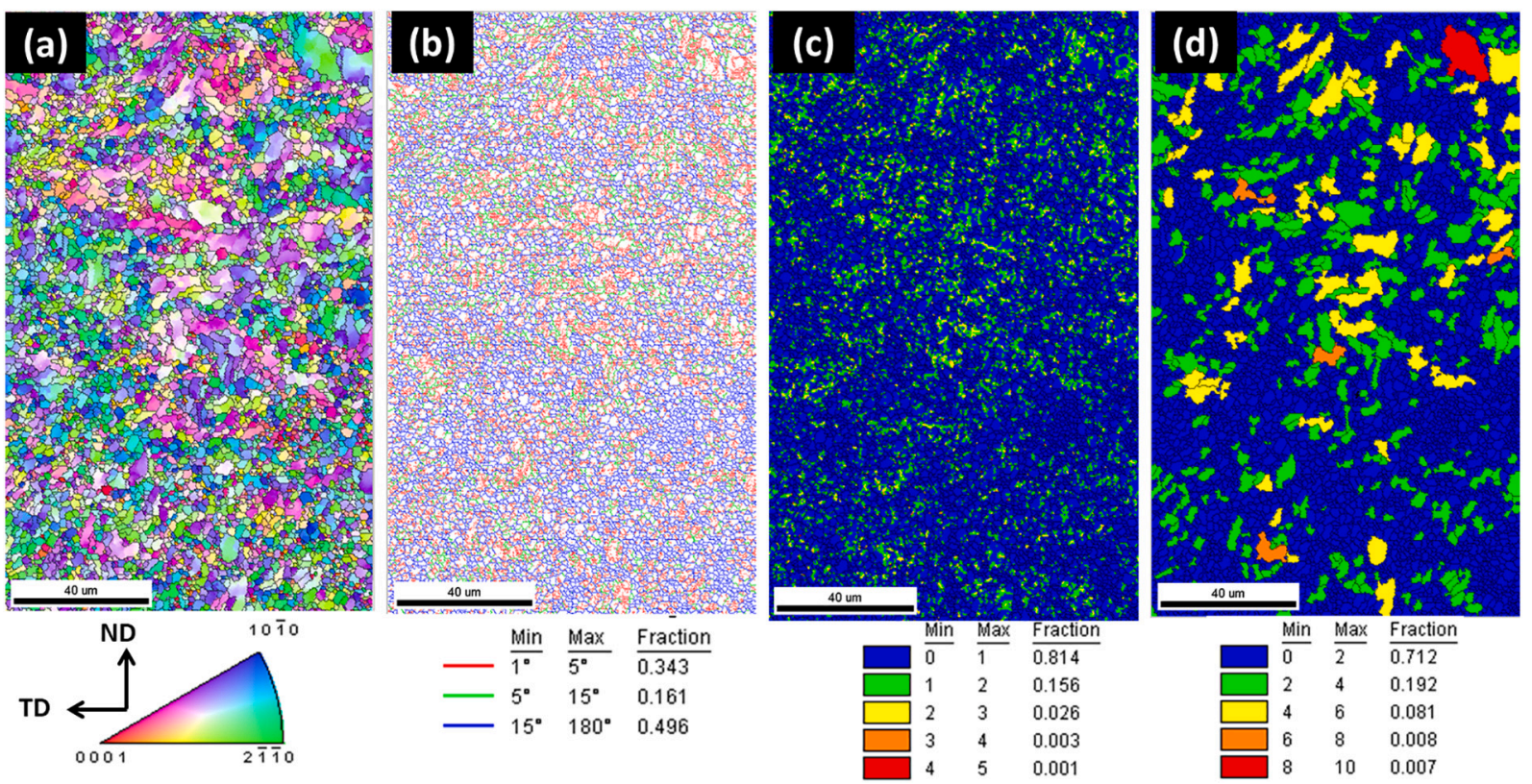

Fig. 5. EBSD generated maps; a) IPF map, b) GBCD map, c) KAM map and d) GOS map with color code and representative tables corresponding to Ti flake.

surrounding material. The conduction of generated heat from the region of peak temperature develops a temperature gradient across the weld nugget. A similar phenomenon occurs for plastic deformation as well. The induced plastic deformation and distribution of temperature rise result in the formation of different weld zones. Each of these zones experiences different levels of stress, strain and temperature.

Furthermore, the crystal structure of $\mathrm{Ti}$ is hexagonal close-packed (HCP) that possesses only four independent slip systems [22]. The available slip systems are insufficient (less than the required five independent slip systems) to accommodate externally induced plastic strain during plastic deformation. Therefore, twinning in Ti is activated as an additional mode of deformation, resulting in plastic deformation without premature failure. The evolution of twins aids in strain hardening rate, thereby increasing plasticity in titanium, which promotes additional plastic deformation without premature failure during deformation. However, it has been stated that twins can accommodate a maximum strain of 0.1 [23] and this operates in Ti up to $400{ }^{\circ} \mathrm{C}$ [24,25]. Beyond this temperature, additional non-basal slip systems come into operation; these enable deformation without twinning [26,27].

Since interfacial friction dictates the temperature rise in the weld nugget and $\mathrm{Al}$ (melting point of $660^{\circ} \mathrm{C}$ ) is present in the nugget zone, the bulk welding temperature never exceeds $660{ }^{\circ} \mathrm{C}$. Therefore, the Ti (melting point of $1660{ }^{\circ} \mathrm{C}$ ) flake is subjected to severe deformation at a lower homologous temperature $\left(\mathrm{T} / \mathrm{T}_{\mathrm{m}}<0.25\right)$. At high strain rates, temperature rise due to plastic deformation is significant in comparison to friction assisted temperature rise. As Ti possesses poor thermal conductivity, the localized heating due to plastic deformation is significant and that leads to the formation of adiabatic shear bands beyond a critical strain [27,28]. Ranc et al. [29] found out that the temperature within the band could exceed $1000^{\circ} \mathrm{C}$. This temperature is much higher than the temperature rise during welding and conventional recrystallization temperature of $\mathrm{Ti}$. For example, recrystallization temperature of $\mathrm{Ti}$ is $775^{\circ} \mathrm{C}$ at a strain rate of $0.001 \mathrm{~s}^{-1}$ [30]. Mayer et al. [30] observed small grains $(0.05-0.3 \mu \mathrm{m}$ in diameter) with HAGB only within the shear bands [28]. A similar characteristic microstructure was observed in the Ti flake of the present study (Fig. 5). Therefore, it can be concluded that the ASB-driven mechanism resulted in the formation of recrystallized microstructure in $\mathrm{Ti}$ in the current investigation. This lead to the formation of different specific zones across the dissimilar weld interface. The evolution of these different zones is schematically illustrated in

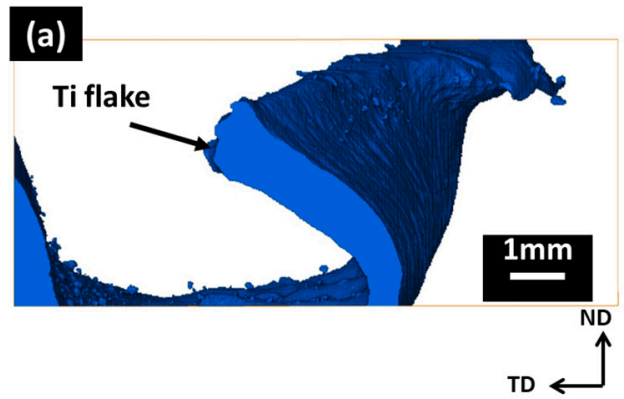

$\mathrm{Ti}$ is represented in blue colour

Ti particles and Al disappear from the image

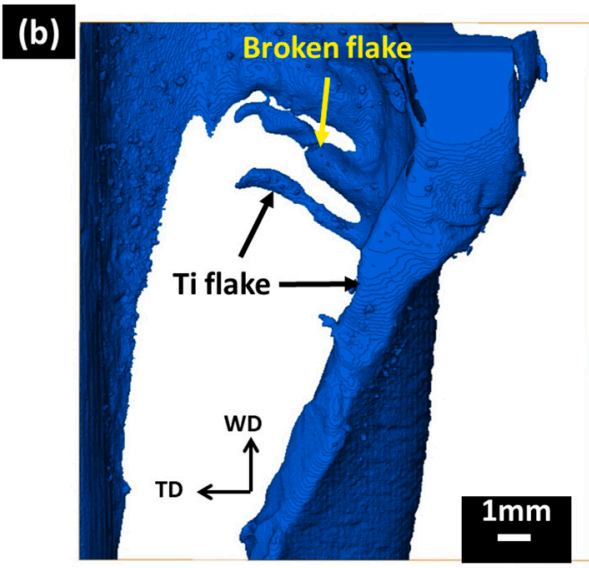

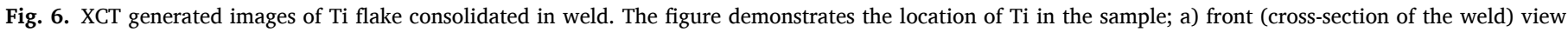
displaying a Ti flake and b) top view showing a large Ti flake and broken Ti flakes in the process of formation of particles. 
Fig. 7(a). The microstructures of the twinning-induced $\mathrm{Ti}$ and recrystallized $\mathrm{Ti}$ are presented in Fig. 4 and Fig. 5 respectively. A gradual microstructure evolution of $\mathrm{Ti}$ from the as-received $\mathrm{Ti}$ to the deformed $\mathrm{Ti}$ (ASB) at the weld interface through the evolution of twins is observed in Fig. 8(a).

The width of the shear band becomes narrow with an increase in plastic deformation [27,31]. Therefore, it is expected to form a narrow width of ASBs at the high strain rate deformation condition that occurs during FSW. The formation of ASBs is schematically shown in Fig. 7(a). The width (d) of the zone at which ASBs are formed significantly depends on the tool offset as it decides the types of particle formation and severity of interaction. The interaction, in turn, decides the value of ' $d$ ' and the width of the strip that is being fragmented from the faying $\mathrm{Ti}$ interface and consolidated on the retreating side. A similar microstructure was noticed in Fig. 2(a). The strain and microstructure inhomogeneity across the ASBs leads to the formation of cracks when titanium is not subjected to recrystallization. At the interface, Ti could contain sharp cracks due to an increase in plastic deformation and reduction in width of ASBs that promotes easy crack formation. Further, this leads to an increase in strain hardening rate and density of ASBs and hence, there is a reduction in critical strain for strain localization and formation of crack [27]. Hence, a high portion of ASBs and micro-cracks are predicted to evolve at the $\mathrm{Ti}$ interface and on the Ti flakes.

During deformation at the time of welding, these cracks propagate along the direction of the applied force that is complex in nature in the case of FSW. This leads to localized deformation and thereafter, fracture in $\mathrm{Ti}$ [32]. Therefore, the weld nugget contains particles with different morphology and sizes. The Ti flake is the biggest particle with a high aspect ratio and carries the signature of deformation and microstructure evolution. The presence of twins and a recrystallized microstructure illustrate the mechanism of microstructure evolution that occurs during deformation, which leads to the fragmentation of Ti to a comparatively finer particle. It was seen that the crack evolves at the location where a substantial fraction of ASBs is noticed. Therefore, these adiabatic shear bands can be considered as paths for easy crack nucleation and propagation, as schematically shown in Fig. 7(a). A number of cracks were noticed at the Ti interface, as shown in Figs. 3 and 8(a). This clearly indicates the formation of ASB-induced cracks in Ti at the weld interface. These cracks and their propagation lead to the formation of large particles. Since this phenomenon originally started at the location of
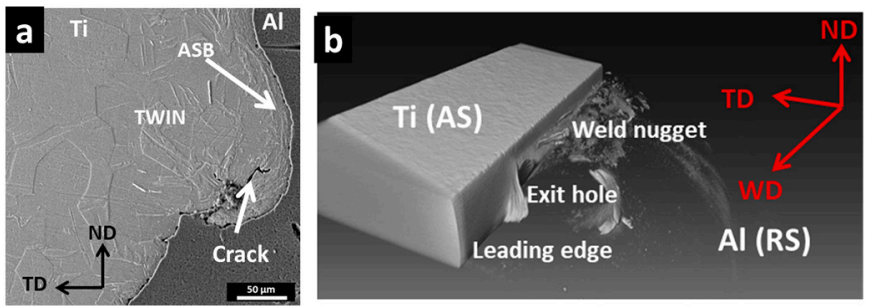

Fig. 8. a) Optical microstructure of the $\mathrm{Al} / \mathrm{Ti}$ weld interface representing a gradual microstructure evolution from the as-received $\mathrm{Ti}$ to the deformed $\mathrm{Ti}$ (ASB) and b) XCT micrograph of the weld exhibiting the formation of large particles near the tool axis and distribution of particles of different sizes in the weld nugget. ND = normal to weld surface, TD = transverse direction and WD $=$ welding direction.

interaction between Ti and the tool, large particles were expected to be seen in the zone of the tool exit hole (Fig. 7(b)). Using XCT, an identical microstructure with large Ti particles was identified, with a reduction in the interaction value ('d' in Fig. 7(b)), as seen in Fig. 8(b). The propagation of one crack along with an ASB aids in the formation of primary particles, and hence, the particle contained with a number of ASBs and fine cracks, as schematically shown in Fig. 7(b). The deformation of large particles during welding develops further ASBs and cracks. The propagation of these cracks helps in the continuous fragmentation of large particles into comparatively fine particles; hence, particles with different sizes were noticed in the nugget zone, as shown in Figs. 2 and 8 (b).

\subsection{Joint formation}

A schematic of the deformation and spreading of Ti across the weld zone is summarized in Fig. 9. The top and side views of material flow are depicted in Figs. 9(a) and 8(b), respectively. At the interface and as flakes, Ti was recrystallized due to the formation of ASBs. The deformed interfaces were further stirred and rotated with the tool. During rotation, the particles containing ASBs were fragmented and lead to the formation of finer particles, as shown in Fig. 9(a). The high velocity of rotation aided in transferring the material in the processed zone. The coarser particles and flakes have a higher mass and hence, swirl away from the
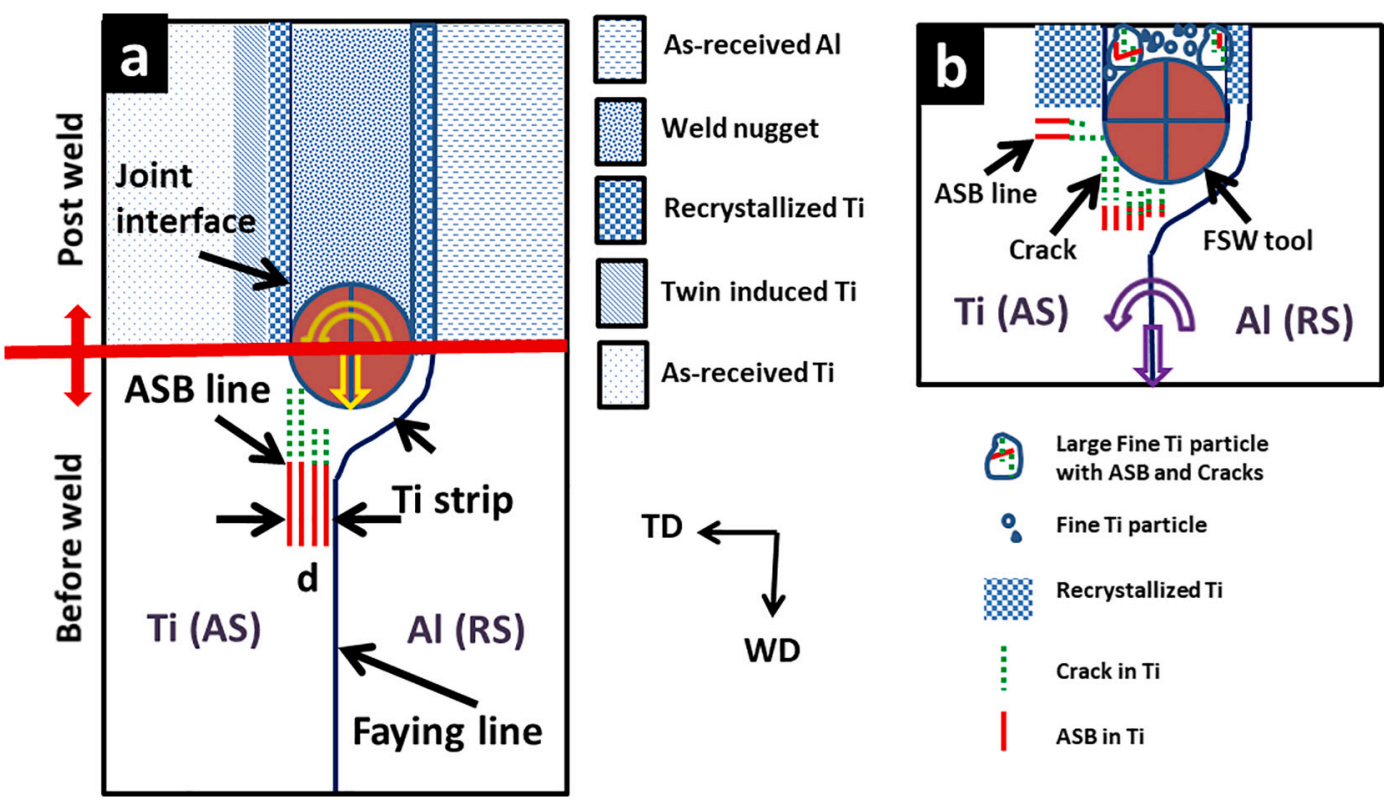

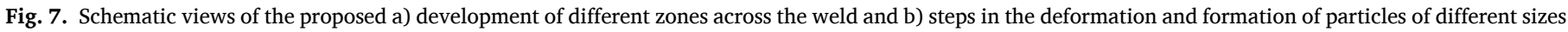
during welding. 

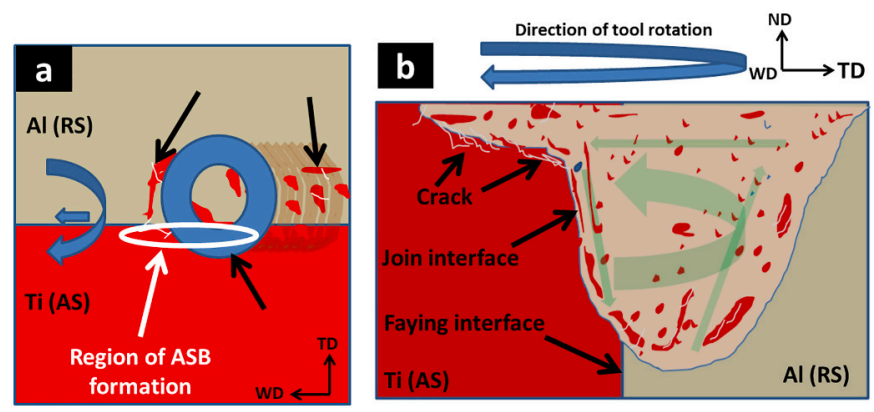

Fig. 9. Schematic views of the proposed deformation and distribution mechanism leading to joint formation; (a) the top view shows the shear deformation of $\mathrm{Ti}$ around the tool and (b) the side view represents the spreading of Ti particles. The arrows indicate the types of material flow.

center of the vortex zone during material flow; therefore, they were consolidated at the edge of the vortex zone. The contact area of the tool shoulder is much higher than that of the tool pin. So, the shoulder of the tool induces a higher heat input and deformation in the processed materials [33]. A downward and upward material flow on the advancing side and the retreating side of the weld were reported by Kumar et al. [7] and Anbukkarasi et al. [34]. A vortex flow pattern in the microstructure has also been reported during the welding of dissimilar materials [34]. The microstructure of this zone is attributed to the dynamic recrystallization of $\mathrm{Al}$ [4]. The vortex zone contains particles that have variations in grain size, layer thickness and hardness [35]. The distribution of particles in this region is homogeneous due to complex mechanical mixing $[17,36]$ and dissimilar material mixing [37], as seen in Fig. 3 (a). The distribution and variation in particle size decide the evolution of local microstructure and final mechanical properties of the weld or/and composite (the weld nugget).

\section{Conclusions}

In the present study, the FSW of commercially pure aluminum and commercially pure titanium was performed and a new technique was used to elucidate the mechanisms associated with the formation and distribution of Ti particle in the nugget zone. To characterize the fragmentation mechanism of $\mathrm{Ti}, \mathrm{Al}$ was dissolved by submerging the weld in a sodium hydroxide solution. The microstructural evolution of the weld was systematically examined in detail. Based on observation and analysis, the following conclusions were drawn:

1. Increase in the degree of shear deformation from base material to weld interface and physical properties of material influence the microstructure evolution across the weld. A lower degree of deformation (strain) in TMAZ results in the evolution of twins due to the availability of less number of active slip system of Ti. In the weld nugget, titanium is subjected to severe deformation, leading to the formation of adiabatic shear bands without twins in Ti in the nugget zone. The existence of a recrystallized microstructure in $\mathrm{Ti}$ is explained as being due to a sudden rise in local temperature in the adiabatic shear bands as compared to the bulk temperature of the weld.

2. Under conditions of severe deformation and lower temperature, the ASBs in Ti become narrow, which reduces the critical strain for crack formation and hence, $\mathrm{Ti}$ in the weld nugget contains sharp microcracks. During welding, these cracks propagate and lead to fracture in the $\mathrm{Ti}$ interface and particles; hence, particles with different morphology and sizes are noticed in the nugget zone.

3. The titanium particles are scattered in the weld nugget. The scatter in their distribution depends on the morphology of the particles. Furthermore, their consolidation depends on location in the weld nugget. A homogenous distribution of particles is noticed at the center of the weld nugget due to complex mechanical mixing. A transition in mixing is attributed to vortex martial flow away from the center of the weld nugget.

4. The material flow also influences the distribution of Ti. The high velocity of rotation transfers material in the weld nugget. The coarser Ti particles and flakes have a higher mass and swirl away from the center of the nugget zone, forming a vortex zone. A high fraction of fine particle is homogeneously distributed in the vortex zone due to severe deformation and complex mechanical mixing.

\section{Data availability statement}

The raw/processed data required to reproduce these findings cannot be shared at this time as the data also forms part of an on-going study. However, I am open to shear any particular data on request.

\section{Declaration of Competing Interest}

The authors declare that they have no known competing financial interests or personal relationships that could have appeared to influence the work reported in this paper.

\section{Acknowledgment}

Authors would like to thank the Defense Research \& Development Organization (DRDO), Department of Science \& Technology (DST), Government of India and Ministry of Education and Science (RMES) of Russian Federation for support and research funding (DSTO1796, The agreement №14.584.21.0023, ID number RFMEFI58417X0023). We would also like to thank IISc, Bengaluru, for providing the research facilities. We express gratitude to Professor Sounak Kumar Choudhury from IIT Kanpur, India, for proofreading.

\section{References}

[1] R.P. Mahto, R. Kumar, S.K. Pal, Characterizations of weld defects, intermetallic compounds and mechanical properties of friction stir lap welded dissimilar alloys, Mater. Charact. 160 (2020) 110115.

[2] A. Kar, B. Vicharapu, Y. Morisada, H. Fujii, Elucidation of interfacial microstructure and properties in friction stir lap welding of aluminium alloy and mild steel, Mater. Charact. 110572 (2020).

[3] A. Kar, S.V. Kailas, S. Suwas, Effect of mechanical mixing in dissimilar friction stir welding of aluminum to titanium with zinc interlayer, Trans. Indian Inst. Metals 72 (2019) 1533-1536.

[4] A. Kar, S.K. Choudhury, S. Suwas, S.V. Kailas, Effect of niobium interlayer in dissimilar friction stir welding of aluminum to titanium, Mater. Charact. 145 (2018) 402-412.

[5] S. Kumar, C. Wu, L. Shi, Intermetallic diminution during friction stir welding of dissimilar $\mathrm{Al} / \mathrm{Mg}$ alloys in lap configuration via ultrasonic assistance, Metall. Mater. Trans. A 51 (2020) 5725-5742.

[6] A. Kar, S. Suwas, S.V. Kailas, Significance of tool offset and copper interlayer during friction stir welding of aluminum to titanium, Int. J. Adv. Manuf. Technol. 100 (2019) 435-443, https://doi.org/10.1007/s00170-018-2682-6.

[7] K. Kumar, S.V. Kailas, Positional dependence of material flow in friction stir welding: analysis of joint line remnant and its relevance to dissimilar metal welding, Sci. Technol. Weld. Join. 15 (2010) 305-311.

[8] T. Singh, S.K. Tiwari, D.K. Shukla, Mechanical and microstructural characterization of friction stir welded AA6061-T6 joints reinforced with nano-sized particles, Mater. Charact. 159 (2020) 110047.

[9] M.M. Moradi, H. Jamshidi Aval, R. Jamaati, S. Amirkhanlou, S. Ji, Effect of SiC nanoparticles on the microstructure and texture of friction stir welded AA2024 AA6061, Mater. Charact. 152 (2019) 169-179.

[10] A. Kar, S.V. Kailas, S. Suwas, Effect of zinc interlayer in microstructure evolution and mechanical properties in dissimilar friction stir welding of aluminum to titanium, J. Mater. Eng. Perform. 27 (2018) 6016-6026, https://doi.org/10.1007/ s11665-018-3697-8.

[11] W. Zhang, Y. Shen, Y. Yan, R. Guo, Dissimilar friction stir welding of 6061 Al to T2 pure $\mathrm{Cu}$ adopting tooth-shaped joint configuration: microstructure and mechanical properties, Mater. Sci. Eng. A 690 (2017) 355-364.

[12] H.C. Madhu, P. Ajay Kumar, C.S. Perugu, S.V. Kailas, Microstructure and mechanical properties of friction stir process derived Al-TiO2 nanocomposite, J. Mater. Eng. Perform. 27 (2018) 1318-1326.

[13] A. Kar, S. Suwas, S.V. Kailas, Two-pass friction stir welding of aluminum alloy to titanium alloy: a simultaneous improvement in mechanical properties, Mater. Sci. Eng. A 733 (2018) 199-210. 
[14] N. Chawla, Y.-L. Shen, Mechanical behavior of particle reinforced metal matrix composites, Adv. Eng. Mater. 3 (2001) 357-370.

[15] L.C. Davis, J.E. Allison, Residual stresses and their effects on deformation, Metall. Trans. A. 24 (1993) 2487-2496.

[17] A. Kar, S. Suwas, S.V. Kailas, Multi-length scale characterization of microstructure evolution and its consequence on mechanical properties in dissimilar friction stir welding of titanium to aluminum, Metall. Mater. Trans. A 50 (2019) 5153-5173.

[18] S.-I. Pyun, S.-M. Moon, Corrosion mechanism of pure aluminium in aqueous alkaline solution, J. Solid State Electrochem. 4 (2000) 267-272.

[19] A. Kar, S. Kailas, S. Suwas, Mechanism of Variation in High-Temperature Grain Stability of Aluminum in Dissimilar Friction Stir Welds, in: Materials Performance and Characterization, 2nd ed., 9, 2020, pp. 262-271.

[20] H. Paul, R. Chulist, P. Bobrowski, K. Perzyński, Ł. Madej, I. Mania, M. Miszczyk, G. Cios, Microstructure and properties of the interfacial region in explosively welded and post-annealed titanium-copper sheets, Mater. Charact. 167 (2020) 110520.

[21] F. Schaff, A. Bachmann, A. Zens, M.F. Zaeh, F. Pfeiffer, J. Herzen, Grating-based Xray dark-field computed tomography for the characterization of friction stir welds: a feasibility study, Mater. Charact. 129 (2017) 143-148.

[22] H. Conrad, Effect of interstitial solutes on the strength and ductility of titanium, Prog. Mater. Sci. 26 (1981) 123-403.

[23] W.J. McG, Tegart, Independent slip systems and ductility of hexagonal polycrystals, Philos. Mag. 9 (1964) 339-341.

[24] E. Yu, I. Kim, D.H. Shin, J. Kim, Deformation mechanism of severely deformed CPtitanium by uniaxial compression test, Mater. Trans. 49 (2008) 38-40.

[25] A.M. Garde, E. Aigeltinger, R.E. Reed-Hill, Relationship between deformation twinning and the stress-strain behavior of polycrystalline titanium and zirconium at 77 K, Metall. Trans. A. 4 (1973) 2461-2468.

[26] M.H. Yoo, Slip, twinning, and fracture in hexagonal close-packed metals, Metall. Trans. A. 12 (1981) 409-418.

[27] S.V. Kailas, Y.V.R.K. Prasad, S.K. Biswas, Microstructural features of flow, Metall. Trans. A. 24 (1993) 2513-2520.
[28] Y. Sun, C. Zhang, H. Feng, S. Zhang, J. Han, W. Zhang, E. Zhao, H. Wang, Dynamic recrystallization mechanism and improved mechanical properties of a near $\alpha$ high temperature titanium alloy processed by severe plastic deformation, Mater. Charact. 163 (2020) 110281.

[29] N. Ranc, L. Taravella, V. Pina, P. Herve, Temperature field measurement in titanium alloy during high strain rate loading-adiabatic shear bands phenomenon, Mech. Mater. 40 (2008) 255-270.

[30] Y.V.R.K. Prasad, T. Seshacharyulu, Processing maps for hot working of titanium alloys, Mater. Sci. Eng. A 243 (1998) 82-88.

[31] S.V. Kailas, Y.V.R.K. Prasad, S.K. Biswas, Flow instabilities and fracture in Ti-6Al4V deformed in compression at 298 K to 673 K, Metall. Mater. Trans. A 25 (1994) 2173-2179.

[32] C. Ran, P. Chen, L. Li, W. Zhang, Y. Liu, X. Zhang, High-strain-rate plastic deformation and fracture behaviour of Ti-5Al-5Mo-5V-1Cr-1Fe titanium alloy at room temperature, Mech. Mater. 116 (2018) 3-10.

[33] A. Kar, D. Yadav, S. Suwas, S.V. Kailas, Role of plastic deformation mechanisms during the microstructural evolution and intermetallics formation in dissimilar friction stir weld, Mater. Charact. 164 (2020) 110371.

[34] R. Anbukkarasi, S.V. Kailas, Influences of shape of the new interfaces and morphology of the intermetallics on mechanical properties of aluminum AA2024-pure copper joints by friction stir welding, Int. J. Adv. Manuf. Technol. 106 (2020) 5071-5083.

[35] N. Nadammal, S.V. Kailas, J. Szpunar, S. Suwas, Microstructure and crystallographic texture evolution during the friction-stir processing of a precipitation-Hardenable aluminum alloy, JOM 67 (2015) 1014-1021.

[36] G. Chen, H. Li, G. Wang, Z. Guo, S. Zhang, Q. Dai, X. Wang, G. Zhang, Q. Shi, Effects of pin thread on the in-process material flow behavior during friction stir welding: a computational fluid dynamics study, Int. J. Mach. Tools Manuf. 124 (2018) 12-21.

[37] C. Yang, C. Wu, L. Shi, Modeling the dissimilar material flow and mixing in friction stir welding of aluminum to magnesium alloys, J. Alloys Compd. 843 (2020) 156021. 